\title{
Global Patterns of Predator Diversity in the Open Oceans
}

\author{
Boris Worm, Marcel Sandow, Andreas Oschlies, Heike K. Lotze, Ransom A. Myers
}

\section{Supporting Online Material}

\section{Materials and Methods}

Diversity data. Tuna and billfish diversity was calculated from 1990-99 Japanese longlining logbook data binned on a global $5^{\circ} \times 5^{\circ}$ grid. These data yielded information on $62,092,629$ individual fish caught on 4.8 billion longline hooks (Table S1). This data set covers the global range of all tuna and billfish species with the exception of coastal areas that are protected by individual countries Exclusive Economic Zones. Countries such as Australia, New Zealand and other Pacific nations however have granted coastal access to Japanese vessels through joint agreements. The rationale for using the 1990s was that fishing techniques did not change significantly during this period, as they had earlier with an increase in average fishing depth during the 1970s and 80s (S1). Furthermore, independent scientific observer data from U.S. and Australian longline fisheries were available to cross-validate the Japanese data for that period (Table S1). Species richness was expressed as the expected number of species from a standardized subsample of size $n$, which is computed as

$$
E\left(S_{n}\right)=\sum_{i=1}^{S}\left[1-\left(\begin{array}{l}
N-m_{i} \\
n
\end{array}\right) /\left(\begin{array}{l}
N \\
n
\end{array}\right)\right]
$$


where $N$ is the total number of individuals in the sample, $S$ is the total number of species in the sample, $m_{i}$ is the number of individuals of species $i$ in the sample $(S 2)$. Species density was calculated as the expected number of species standardized to $k=1000$ hooks. In this case, the number of individuals per 1000 hooks determines $n$, and hence diversity is also dependent on the abundance of species. We chose 50 individuals and 1000 hooks as standardized subsample sizes because these correspond roughly to the average number of individuals and hooks sampled by a single longlining set. Using other subsample sizes $(n=20,100,500, k=500,2000,5000)$ did not change diversity patterns. Similarly, checking robustness by randomly deleting single species and re-calculating diversity did not change the results. This means that diversity patterns were not driven by any particular species.

Using the same methods as outlined above, total predator diversity was derived from U.S. and Australian scientific observer data as supplied by the U.S. National Marine Fishery Service (NOAA/NMFS) in the Northwest Atlantic (Atlantic observer data, since 1991, $\mathrm{N}=1962$ longline sets) and around Hawaii (Hawaiian observer data, since 1994, N= 3290 sets), and by the Australian Fisheries Management Authority (AFMA; Australian observer data, since 1991, $\mathrm{N}=3127$ sets). These data yielded information on 439,136 individual fish, turtles, mammals and birds caught on 12.06 million longline hooks (Table S1). Foraminiferan zooplankton data as used by Rutherford et al. $(S 3)$ were retrieved from the Brown University Foraminifera Database, and binned across a global $5^{\circ} \times 5^{\circ}$ grid. Diversity was expressed as species richness per sample. Regression analyses were reported for the Atlantic foraminiferan data (Fig. 1G), as only the Atlantic data were deemed reliable 
enough for analysis $(S 3)$. Yet the relationship between the global foraminiferan and tuna and billfish data set was equally strong $(r=0.63, P<0.0001)$.

Oceanographic analysis. To obtain global coverage at a spatial resolution high enough to resolve mesoscale variability of the upper ocean, we based our analysis of oceanographic variables on fine-scale satellite data covering 1998-2002. Five-daily maps of sea surface temperature (SST) at $0.5^{\circ}$ resolution were provided by the NOAA/NASA AVHRR Oceans Pathfinder project. Error estimates for this data set range from $0.3-0.5^{\circ} \mathrm{C}$. Weekly maps of satellite-derived sea surface height $(\mathrm{SSH})$ anomalies at $0.35^{\circ}$ resolution were derived from the TOPEX/Poseidon altimeter provided by the French Aviso. SSH anomalies were used to calculate eddy kinetic energy according to (S4). Weekly surface chlorophyll $a$ fields at $0.35^{\circ}$ resolution were derived from SeaWiFS satellite ocean colour data. The error level of these data is estimated as 35\%. Oxygen data at $100 \mathrm{~m}$ depth (corresponding to the average depth of a longline) were derived from the Levitus data set (NOAA National Oceanographic Data Center, Silver Springs, Maryland). Bathymetric data at $0.08^{\circ}$ resolution were derived from the ETOPO5 data set (NOAA National Geophysical Data Center, Boulder, Colorado). Spatial gradients in SST $\left({ }^{\circ} \mathrm{C} \mathrm{km}^{-1}\right)$, chlorophyll $a\left(\mathrm{mg} \mathrm{m}^{-3} \mathrm{~km}^{-}\right.$

$\left.{ }^{1}\right)$, and bathymetry $\left(\mathrm{m} \mathrm{km}^{-1}\right)$ were estimated by calculating the maximum absolute slope of each data point (at original resolution of $0.5^{\circ}, 0.35^{\circ}$, or $0.08^{\circ}$, respectively) to its eight surrounding points. Slopes were subsequently averaged across $5^{\circ} \times 5^{\circ}$ grid cells. Alternatively, we calculated frontal density from slope data by using a lower cut-off of 0.01 or $0.02{ }^{\circ} \mathrm{C} \mathrm{km}^{-1}(S 5)$. This gave similar results as the mean slope which we report here. We 
then fitted spatial regression models to these data in an attempt to predict diversity from oceanographic data. Eddy kinetic energy, chlorophyll $a$ and depth gradient data were logtransformed to improve linearity. Spatial regression models accounted for possible spatial dependence among cells by using a conditional autoregressive model (S6). The spatial covariance between two $5^{\circ} \times 5^{\circ}$ cells $y_{i}$ and $y_{j}$ was assumed to decline with distance $d$ in an anisotropic exponential decay function, such that $\operatorname{cov}\left(y_{i}, y_{j}\right)=\sigma^{2} \exp -\left(\theta_{1} d_{i, j, 1}+\theta_{2} d_{i, j, 2}\right)$

where $\theta_{1}$ describes the latitudinal and $\theta_{2}$ the longitudinal covariance parameter, $d_{i, j, 1}$ is the latitudinal distance and $d_{i, j, 2}$ the longitudinal distance between cells. Covariance parameters were estimated from the data using maximum likelihood (Procedure MIXED in SAS V.8). Cells from different oceans were assumed independent. We first fitted this model to the 1990 s data, and then confirmed its robustness by fitting it to diversity and SST data from previous decades (1960-99). In these cases we used extended reconstructed sea surface temperature data (ERSST) as provided by the NOAA-CIRES climate diagnostic center (University of Colorado, Boulder, CO, USA). These analyses produced very consistent results across decades (Table S3).

Historic trends in diversity. Using mixed effects models we estimated long-term trends and short-term variability in tuna and billfish diversity and examined relationships with fishing and climate for each ocean. Trends in tuna and billfish diversity over time were estimated from 1952-99 Japanese longlining data. Using recently derived correction factors 
for each species $(S 7)$, we first standardized Japanese longline data for historic changes in fishing practices, particularly the increase in longline depth during the 1970s and 1980s to target deeper-swimming species such as bigeye tuna (Thunnus obesus). Species richness and species density by year were calculated from these data by rarefaction as outlined above. The resulting data sets are displayed in Movies S1 and S2, respectively. From these data sets we estimated trends in species richness and species density for each ocean using linear mixed effects models that accounted for any changes in the coverage and seasonality of fished cells. We fitted the model

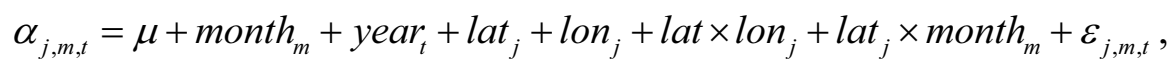

where $\alpha_{j, m, t}$ refers to diversity (species richness or species density) in cell $j$ (defined by its latitude and longitude), month $m$, year $t$, and where $\mu$ describes the mean across all cells, months and years, and $\varepsilon_{i, k, l, m}$ the random error. Year and month were fixed categorical effects, while the other terms were modeled as random effects with normal distribution, zero mean and variances $\sigma_{j}^{2}, \sigma_{m}^{2}$, and $\sigma_{t}^{2}$, respectively. Alternative analyses treating latitude and longitude as fixed effects yielded similar results. For further analysis we calculated the estimated least square means for the year effects in diversity $D_{t}$ across each ocean. The first years in the Atlantic (1956-60) were excluded due to low sample size and latitudinal coverage.

Long-term changes in diversity were plotted against total catches of tuna and billfish (all gear types combined), compiled from the Food and Agriculture Organization (FAO) database. Year-to-year variation in diversity, i.e. the first difference in species richness $\Delta_{t}=D_{t+1}-D_{t}$ was calculated from the mixed effects model output for each ocean. Those 
time series were initially correlated at zero lag with the multivariate El Niño Southern Oscillation (ENSO) index (Dec-Mar average) provided by the NOAA-CIRES climate diagnostic center (University of Colorado, Boulder, CO, USA). Longer time lags attenuated the correlation. Similar analyses were performed using the Pacific Decadal Oscillation Index $(S 8)$ supplied by the Joint Institute for the Study of the Atmosphere and the Ocean (Washington University, Seattle, WA, USA), the North Atlantic Oscillation Index (S9) supplied by the Climate Research Unit (University of East Anglia, Norwich, UK), and the Indian Ocean Dipole Index (S10) supplied by the Japanese Agency for Marine-Earth Science and Technology (Tokyo, Japan). Here, temporal autocorrelation was effectively removed by first-differencing, as confirmed by the Durbin-Watson test. Spatial variation among cells in response to ENSO across the Pacific was estimated using a mixed effects model for the first difference in diversity

$$
\Delta_{j, t}=\beta E N S O_{t}+\beta_{j} E_{N S O}+\varepsilon_{j, t},
$$

where $\beta$ is the slope parameter that describes the mean rate of change in diversity with $\mathrm{ENSO}, \beta_{j}$ is the random slope component for cell $j$, which is assumed normal with zero mean and variance $\sigma_{\beta}^{2}$, and $\varepsilon_{i, j}$ is the random error also assumed normal with zero mean and variance $\sigma^{2}$. Best linear unbiased predictions for $\beta_{j}$ were calculated and plotted for each cell to describe the local variation in the response in the change in diversity to the ENSO index. Similar analysis was carried out for the change in log catch rates for each species. Simple linear correlations of $\Delta_{j, t}$ with ENSO yielded index very similar results. 


\section{References}

S1. Y. Uozumi, H. Nakano, in Collective Volume of Scientific Papers. Report of the second ICCAT Billfish Workshop. (International Commission for the Conservation of Atlantic Tunas, Madrid, Spain, 1996) pp. 233-243.

S2. N. J. Gotelli, G. R. Graves, Null Models in Ecology (Smithsonian Institution Press, Washington D.C., 1996).

S3. S. Rutherford, S. D'Hondt, W. Prell, Nature 400, 749-753 (1999).

S4. A. Oschlies, V. Garçon, Nature 394, 266-269 (1998).

S5. P. Etnoyer, D. Canny, B. Mate, L. Morgan, Oceanography 17, 90-101 (2004).

S6. N. A. C. Cressie, Statistics for Spatial Data (John Wiley \& Sons, New York, 1993).

S7. P. Ward, R. A. Myers, Can. J. Fish. Aquat. Sci. 62, 1130-1142 (2005).

S8. N. J. Mantua, S. R. Hare, Y. Zhang, J. M. Wallace, R. C. Francis, Bull. Am. Meteorol. Soc. 78, 1069-1079 (1997).

S9. J. W. Hurrell, Science 269, 676-679 (1995).

S10. N. H. Saji, B. N. Goswami, P. N. Vinayachandran, T. Yamagata, Nature 401, 360 - 363 (1999). 
Table S1. Sample sizes of species identified in the Japanese and regional observer data sets 1990-99

\begin{tabular}{|c|c|c|c|c|c|c|}
\hline Category & Common Name & Scientific Name & $\begin{array}{l}\text { Global } \\
\text { Japanese }\end{array}$ & $\begin{array}{l}\text { Atlantic } \\
\text { observer }\end{array}$ & $\begin{array}{l}\text { Hawaii } \\
\text { observer }\end{array}$ & $\begin{array}{l}\text { Australia } \\
\text { observer }\end{array}$ \\
\hline \multirow[t]{12}{*}{ Billfish } & Atlantic blue marlin & Makaira nigricans & 106944 & 554 & - & - \\
\hline & Black marlin & Makaira indica & 40116 & - & 37 & 251 \\
\hline & Indo-Pacific blue marlin & Makaira mazara & 997978 & - & 1334 & 295 \\
\hline & Longbill spearfish & Tetrapturus pfluegeri & - & 72 & - & - \\
\hline & Marlin & Makaira sp. & - & - & - & 4 \\
\hline & Roundscale spearfish & Tetrapturus georgei & - & 8 & - & - \\
\hline & Sailfish & Istiophorus platypterus & 96265 & 514 & 104 & 203 \\
\hline & Shortbill spearfish & Tetrapturus angustirostris & - & - & 2146 & 1090 \\
\hline & Spearfish & Tetrapturus sp. & 94582 & 67 & - & - \\
\hline & Striped marlin & Tetrapturus audax & 1152396 & - & 3640 & 1505 \\
\hline & Swordfish & Xiphias gladius & 2310633 & 28621 & 17121 & 3686 \\
\hline & White marlin & Tetrapturus albidus & 32060 & 762 & - & - \\
\hline \multirow[t]{12}{*}{ Tuna } & Albacore tuna & Thunnus alalunga & 13853138 & 1020 & 14669 & 48010 \\
\hline & Atlantic bluefin tuna & Thunnus thynnus & 298617 & 396 & - & - \\
\hline & Bigeye tuna & Thunnus obesus & 26304855 & 3039 & 13007 & 6485 \\
\hline & Blackfin tuna & Thunnus atlanticus & - & 131 & - & - \\
\hline & Bullet tuna & Auxis rochei rochei & - & - & 1 & - \\
\hline & Little tuna & Euthynnus alletteratus & - & 66 & - & - \\
\hline & Kawakawa & Euthynnus affinis & - & - & 4 & 1 \\
\hline & Pacific bluefin tuna & Thunnus orientalis & 13398 & - & 72 & 12 \\
\hline & Skipjack tuna & Katsuwonus pelamis & 135394 & 42 & 2097 & 1507 \\
\hline & Slender tuna & Allothunnus fallai & - & - & - & 72 \\
\hline & Southern bluefin tuna & Thunnus maccoyii & 1434572 & - & - & 31231 \\
\hline & Yellowfin tuna & Thunnus albacares & 15221681 & 3208 & 5651 & 23244 \\
\hline \multirow[t]{24}{*}{ Other bony fish } & Amberjack & Seriola sp. & - & 1 & - & - \\
\hline & Atlantic cutlassfish & Trichiurus lepturus & - & 2 & - & - \\
\hline & Banded rudderfish & Seriola zonata & - & - & - & - \\
\hline & Barracouta & Thyrsites sp. & - & - & - & 100 \\
\hline & Barracuda & Sphyraenidae & - & 108 & - & - \\
\hline & Bigeye cigarfish & Cubiceps sp. & - & 55 & - & - \\
\hline & Bigeye scad & Selar crumenophthalmus & - & - & 7 & - \\
\hline & Black sea bass & Centropristis striata & - & 1 & - & - \\
\hline & Blackfin snapper & Lutjanus buccanella & - & 1 & - & - \\
\hline & Blue grenadier & Macruronus novaezelandiae & - & - & - & 11 \\
\hline & Bluefish & Pomatomus saltatrix & - & 44 & - & - \\
\hline & Bonito & Sarda sarda & - & 19 & - & - \\
\hline & Butterfly mackerel & Gasterochisma melampus & - & - & - & 1440 \\
\hline & Chub mackerel & Scomber japonicus & - & 6 & 1 & - \\
\hline & Cobia & Rachycentron canadum & - & 1 & - & - \\
\hline & Common dolphinfish & Coryphaena hippurus & - & 5070 & 14563 & 822 \\
\hline & Common sunfish & Mola ramsayi & - & - & 126 & 925 \\
\hline & Conger eel & Congridae & - & - & - & 1 \\
\hline & Crestfish & Lophotus lacepede & - & - & 23 & - \\
\hline & Cutlassfishes & Trichiuridae & - & 111 & - & - \\
\hline & Dagger pomfret & Taractes rubescens & - & - & 51 & - \\
\hline & Dealfish & Trachipteridae & - & 1 & 4 & - \\
\hline & Deep sea trevalla & Hyperoglyphe antarctica & - & - & - & 11 \\
\hline & Escolar & Lepidocybium flavobrunneum & - & 1253 & 1359 & 4010 \\
\hline
\end{tabular}




\begin{tabular}{|c|c|c|c|c|c|c|}
\hline & Flying Fish & Exocoetidae & - & - & 1 & 3 \\
\hline & Frigate mackerel & Auxis thazard & - & 4 & - & - \\
\hline & Gemfish & Rexea solandri & - & - & - & 16 \\
\hline & Goosefish & Lophiidae & - & 1 & - & - \\
\hline & Great barracuda & Sphyraena barracuda & - & - & 235 & 111 \\
\hline & Jack & Caranx sp. & - & 1 & - & - \\
\hline & King mackerel & Scomberomorus cavalla & - & 5 & - & - \\
\hline & Lancetfish & Alepisaurus sp. & - & 1038 & 7453 & - \\
\hline & Long-finned bream & Taractichthys longipinnis & - & - & - & 742 \\
\hline & Long-nosed lancet fish & Alepisaurus ferox & - & - & - & 7884 \\
\hline & Louvar & Luvarus imperialis & - & - & 1 & - \\
\hline & Oarfish & Regalecus glesne & - & - & 6 & - \\
\hline & Oilfish & Ruvetus pretiosus & - & 404 & 623 & 4960 \\
\hline & Opah & Lampris guttatus & - & 1 & 1207 & 990 \\
\hline & Pacific pomfret & Brama japonica & - & - & 237 & - \\
\hline & Pelagic puffer & Lagocephalus lagocephalus & - & - & 32 & - \\
\hline & Pomfret & Bramidae & - & 22 & - & 202 \\
\hline & Puffer & Tetraodontidae & - & 45 & - & - \\
\hline & Rainbow runner & Elagatis bipinnulatus & - & 1 & 6 & - \\
\hline & Ray's Bream & Brama brama & - & - & - & 27278 \\
\hline & Remora & Echeneidae & - & 8 & 9373 & - \\
\hline & Ribbonfishes & Trachipteridae & - & - & - & 114 \\
\hline & Rudderfish & Centrolophus niger & - & - & - & 239 \\
\hline & Short-nosed lancet fish & Alepisaurus brevirostris & - & - & - & 723 \\
\hline & Sickle pomfret & Taractichthys steindachneri & - & - & 1659 & - \\
\hline & Slender barracuda & Sphyraena jello & - & - & - & 185 \\
\hline & Slender sunfish & Ranzania laevis & - & - & 43 & - \\
\hline & Snake mackerel & Gempylus serpens & - & - & 2683 & 50 \\
\hline & Southern ray's bream & Brama sp. & - & - & - & 91 \\
\hline & Sunfish & Mola sp. & - & 101 & - & - \\
\hline & Triggerfish & Balistidae & - & 3 & - & - \\
\hline & Tripletail & Lobotes surinamensis & - & 1 & - & - \\
\hline & Wahoo & Acanthocybium solandri & - & 192 & 1233 & 474 \\
\hline & Yellowtail kingfish & Seriola lalandi & - & - & 1 & 57 \\
\hline Turtles & Green turtle & Chelonia mydas & - & 12 & 10 & - \\
\hline & Hawksbill turtle & Eretmochelys imbricata & - & 3 & - & - \\
\hline & Leatherback turtle & Dermochelys coriacea & - & 164 & 44 & - \\
\hline & Loggerhead turtle & Caretta caretta & - & 287 & 166 & - \\
\hline & Olive ridley turtle & Lepidochelys olivacea & - & - & 36 & - \\
\hline Sharks and rays & Atlantic sharpnose shark & Rhizoprionodon terraenovae & - & 15 & - & - \\
\hline & Bigeye thresher shark & Alopias superciliosus & - & 205 & 591 & - \\
\hline & Bignose shark & Carcharhinus altimus & - & 30 & 26 & - \\
\hline & Blacktip shark & Carcharhinus limbatus & - & 70 & - & - \\
\hline & Blue shark & Prionace glauca & - & 26757 & 33346 & 37310 \\
\hline & Bronze whaler shark & Carcharhinus brachyurus & - & - & - & 202 \\
\hline & Bull shark & Carcharhinus leucas & - & 26 & - & - \\
\hline & Common thresher shark & Alopias vulpinus & - & 37 & - & 144 \\
\hline & Cookie cutter shark & Isistius brasiliensis & - & 2 & 18 & 62 \\
\hline & Crocodile shark & Pseudocarcharias kamoharai & - & 156 & 170 & 921 \\
\hline & Dogfish & Squalidae & - & 1 & - & 118 \\
\hline & Dusky shark & Carcharhinus obscurus & - & 649 & 26 & 313 \\
\hline & Galapagos shark & Carcharhinus galapagensis & - & - & 5 & - \\
\hline
\end{tabular}




\begin{tabular}{|c|c|c|c|c|c|c|}
\hline & Great hammerhead shark & Sphyrna mokarran & - & 49 & - & - \\
\hline & Great white shark & Carcharodon carcharias & - & - & 3 & - \\
\hline & Hammerhead sp. & Sphyrna sp. & - & 111 & 4 & 57 \\
\hline & Lemon shark & Negaprion brevirostrus & - & 1 & - & - \\
\hline & Longfin mako shark & Isurus paucus & - & 47 & 14 & 4 \\
\hline & Mako sp. & Isurus sp. & - & 238 & 10 & - \\
\hline & Manta ray & Mobulidae & - & - & 12 & 22 \\
\hline & Night shark & Carcharhinus signatus & - & 310 & - & - \\
\hline & Nurse shark & Ginglymostoma cirratum & - & 1 & - & - \\
\hline & Oceanic whitetip shark & Carcharhinus longimanus & - & 278 & 1067 & 246 \\
\hline & Pelagic stingray & Pteroplatytrygon violacea & - & 39 & 2851 & 1906 \\
\hline & Pelagic thresher shark & Alopias pelagicus & - & - & - & 1 \\
\hline & Porbeagle shark & Lamna nasus & - & 14 & - & 2421 \\
\hline & Ray & Chondrichthyes & - & 1452 & - & - \\
\hline & Reef shark & Carcharinus perezii & - & 7 & - & - \\
\hline & Salmon shark & Lamna ditropis & - & - & 65 & - \\
\hline & Sandbar shark & Carcharhinus plumbeus & - & 188 & 25 & 1 \\
\hline & Scalloped hammerhead shark & Sphyrna lewini & - & 356 & - & - \\
\hline & School shark & Galeorhinus galeus & - & - & - & 224 \\
\hline & Shortfin mako shark & Isurus paucus & - & 1051 & 519 & 1516 \\
\hline & Silky shark & Carcharhinus falciformis & - & 1789 & 183 & 11 \\
\hline & Smooth hammerhead shark & Sphyrna zygaena & - & 4 & 17 & - \\
\hline & Spinner shark & Carcharhinus brevipinna & - & 12 & - & - \\
\hline & Spiny dogfish & Squalus acanthias & - & 18 & - & - \\
\hline & Thresher sp. & Alopias sp. & - & 15 & 111 & 257 \\
\hline & Tiger shark & Galeocerdo cuvieri & - & 284 & 6 & 61 \\
\hline & Velvet dogfish & Zameus squamulosus & - & - & - & 236 \\
\hline Mammals & Australian fur seal & Arctocephalus pusillus & - & - & - & 3 \\
\hline & Beaked whale & Ziphiidae & - & 1 & - & - \\
\hline & Bottlenose dolphin & Tursiops truncatus & - & 4 & 2 & - \\
\hline & Dolphin & Stenella sp. & - & 1 & 2 & 1 \\
\hline & False killer whale & Pseudorca crassidens & - & - & 2 & - \\
\hline & Killer whale & Orcinus orca & - & 1 & - & - \\
\hline & Pantropic spotted dolphin & Stenella attenuata & - & 2 & - & - \\
\hline & Pilot whale sp. & Globicephala sp. & - & 12 & - & - \\
\hline & Risso's dolphin & Grampus griseus & - & 4 & 6 & - \\
\hline & Short spinner dolphin & Stenella clymene & - & 1 & - & - \\
\hline & Shortfin pilot whale & Globicephala macrorhynchus & - & 1 & - & - \\
\hline & Sperm whale & Physeter macrocephalus & - & - & 1 & - \\
\hline & Whale & Cetacea & - & - & 5 & 3 \\
\hline Seabirds & Albatross sp. & Diomedeidae sp. & - & - & - & 261 \\
\hline & Black-footed albatross & Phoebastria nigripes & - & - & 624 & - \\
\hline & Gull & Larinae & - & 1 & - & - \\
\hline & Laysan albatross & Diomedea immutabilis & - & - & 437 & - \\
\hline & Other seabirds & Aves & - & 12 & 4 & 791 \\
\hline & Petrel sp. & Procellariidae & - & - & - & 73 \\
\hline Number of & & & 62092629 & 81718 & 141218 & 216200 \\
\hline Number of & & & 15 & 90 & 71 & 67 \\
\hline Number of & & & - & 1962 & 3290 & 3127 \\
\hline Number of & $(1000)$ & & 4801751 & 1116 & 3835 & 7109 \\
\hline Mean hook & & & - & 569 & 1166 & 2273 \\
\hline Mean indiv & er 1000 hooks & & 12.9 & 73.2 & 36.8 & 30.4 \\
\hline
\end{tabular}


Table S2. Mixed model results for trends in diversity over time

\begin{tabular}{|c|c|c|c|c|c|c|c|c|}
\hline \multirow{2}{*}{$\begin{array}{l}\text { Variable } \\
\text { Fixed effects }\end{array}$} & \multicolumn{4}{|c|}{ Species richness Atlantic Ocean } & \multicolumn{4}{|c|}{ Species density Atlantic Ocean } \\
\hline & $\mathrm{df}$ (num.) & df (denom.) & $F$ & $P$ & $\mathrm{df}$ (num.) & $\mathrm{df}$ (denom.) & $F$ & $P$ \\
\hline Month & 11 & 211 & 2.8 & 0.0024 & 11 & 211 & 2.0 & 0.0295 \\
\hline Year & 43 & 22000 & 50.0 & $<0.0001$ & 43 & 22000 & 234.5 & $<0.0001$ \\
\hline Covariance parameters & estimate & s.e. & $Z$ & $P$ & estimate & s.e. & $Z$ & $P$ \\
\hline Latitude (Lat) & 0.766 & 0.242 & 3.2 & 0.0008 & 0.405 & 0.127 & 3.2 & 0.0007 \\
\hline Longitude (Lon) & 0.343 & 0.110 & 3.1 & 0.0009 & 0.314 & 0.097 & 3.2 & 0.0006 \\
\hline Lat $x$ Lon & 0.147 & 0.015 & 9.6 & $<0.0001$ & 0.097 & 0.010 & 9.6 & $<0.0001$ \\
\hline Lat $x$ Month & 0.033 & 0.004 & 7.7 & $<0.0001$ & 0.020 & 0.003 & 7.7 & $<0.0001$ \\
\hline Residual & 0.645 & 0.006 & 105.5 & $<0.0001$ & 0.422 & 0.004 & 105.5 & $<0.0001$ \\
\hline Variable & \multicolumn{4}{|c|}{ Species richness Indian Ocean } & \multicolumn{4}{|c|}{ Species density Indian Ocean } \\
\hline Fixed effects & $\mathrm{df}$ (num.) & df (denom.) & $F$ & $P$ & df (num.) & $\mathrm{df}$ (denom.) & $F$ & $P$ \\
\hline Month & 11 & 135 & 4.6 & $<0.0001$ & 11 & 135 & 4.1 & $<0.0001$ \\
\hline Year & 46 & 28000 & 27.6 & $<0.0001$ & 46 & 28000 & 439.7 & $<0.0001$ \\
\hline Covariance parameters & estimate & s.e. & $Z$ & $P$ & estimate & s.e. & $Z$ & $P$ \\
\hline Latitude (Lat) & 1.975 & 0.769 & 2.6 & 0.0051 & 1.061 & 0.414 & 2.6 & 0.0052 \\
\hline Longitude (Lon) & 0.134 & 0.048 & 2.8 & 0.0028 & 0.098 & 0.034 & 2.9 & 0.0020 \\
\hline Lat $x$ Lon & 0.139 & 0.017 & 8.3 & $<0.0001$ & 0.084 & 0.010 & 8.4 & $<0.0001$ \\
\hline Lat $x$ Month & 0.025 & 0.004 & 6.4 & $<0.0001$ & 0.011 & 0.002 & 5.9 & $<0.0001$ \\
\hline Residual & 0.673 & 0.006 & 119.1 & $<0.0001$ & 0.391 & 0.003 & 119.1 & $<0.0001$ \\
\hline Variable & \multicolumn{4}{|c|}{ Species richness Pacific Ocean } & \multicolumn{4}{|c|}{ Species density Pacific Ocean } \\
\hline Fixed effects & $\mathrm{df}$ (num.) & df (denom.) & $F$ & $P$ & df (num.) & df (denom.) & $F$ & $P$ \\
\hline Month & 11 & 205 & 1.9 & 0.0454 & 11 & 205 & 3.8 & $<0.0001$ \\
\hline Year & 47 & 93000 & 91.1 & $<0.0001$ & 47 & 93000 & 377.1 & $<0.0001$ \\
\hline Covariance parameters & estimate & s.e. & $Z$ & $P$ & estimate & s.e. & $Z$ & $P$ \\
\hline Latitude (Lat) & 0.846 & 0.291 & 2.9 & 0.0018 & 0.623 & 0.202 & 3.1 & 0.0010 \\
\hline Longitude (Lon) & 0.026 & 0.012 & 2.2 & 0.0150 & 0.065 & 0.020 & 3.2 & 0.0006 \\
\hline Lat $x$ Lon & 0.177 & 0.013 & 13.3 & $<0.0001$ & 0.088 & 0.007 & 13.1 & $<0.0001$ \\
\hline Lat $x$ Month & 0.111 & 0.012 & 9.3 & $<0.0001$ & 0.040 & 0.005 & 8.1 & $<0.0001$ \\
\hline Residual & 0.535 & 0.002 & 215.8 & $<0.0001$ & 0.316 & 0.001 & 215.7 & $<0.0001$ \\
\hline
\end{tabular}


Table S3. Spatial regression models for depth-corrected decadal data 1960-1999

\begin{tabular}{|c|c|c|c|c|c|c|c|c|}
\hline \multirow[t]{2}{*}{ Variable } & \multicolumn{4}{|c|}{ Species richness 1960-69 } & \multicolumn{4}{|c|}{ Species density 1960-69 } \\
\hline & coefficient & s.e. & $t$ & $P$ & coefficient & s.e. & $t$ & $P$ \\
\hline Intercept & 2.510 & 0.862 & 2.9 & 0.1005 & 1.551 & 0.724 & 2.1 & 0.1655 \\
\hline SST & -0.453 & 0.153 & -3.0 & 0.0032 & -0.313 & 0.128 & -2.5 & 0.0143 \\
\hline$(\mathrm{SST})^{2}$ & 0.034 & 0.009 & 3.6 & 0.0004 & 0.028 & 0.008 & 3.6 & 0.0004 \\
\hline$(\mathrm{SST})^{3}$ & -0.001 & 0.0002 & -3.2 & 0.0015 & -0.001 & 0.0001 & -3.6 & 0.0004 \\
\hline SST gradient & 52.438 & 12.044 & 4.4 & $<0.0001$ & 37.586 & 10.304 & 3.7 & 0.0003 \\
\hline Dissolved oxygen & 0.081 & 0.047 & 1.7 & 0.0853 & 0.090 & 0.041 & 2.2 & 0.0276 \\
\hline Covariance parameters & $\theta_{1}$ & $\theta_{2}$ & & $\sigma^{2}$ & $\theta_{1}$ & $\theta_{2}$ & & \\
\hline Estimate & 0.227 & 0.042 & & 0.473 & 0.191 & 0.046 & & \\
\hline Likelihood ratio test & $d f=2$ & $X^{2}=248.4$ & & $<0.0001$ & $d f=2$ & $X^{2}=272.2$ & & 001 \\
\hline \multirow[t]{2}{*}{ Variable } & \multicolumn{4}{|c|}{ Species richness 1970-79 } & \multicolumn{4}{|c|}{ Species density 1970-79 } \\
\hline & coefficient & s.e. & $t$ & $P$ & coefficient & s.e. & $t$ & $P$ \\
\hline Intercept & 2.763 & 0.869 & 3.2 & 0.0863 & 1.119 & 0.681 & 1.6 & 0.2421 \\
\hline SST & -0.543 & 0.154 & -3.5 & 0.0004 & -0.340 & 0.121 & -2.8 & 0.0050 \\
\hline$(\mathrm{SST})^{2}$ & 0.043 & 0.009 & 4.6 & $<0.0001$ & 0.030 & 0.007 & 4.1 & $<0.0001$ \\
\hline$(\mathrm{SST})^{3}$ & -0.001 & 0.0002 & -4.5 & $<0.0001$ & -0.001 & 0.0001 & -4.2 & $<0.0001$ \\
\hline SST gradient & 31.861 & 13.684 & 2.3 & 0.0201 & 28.938 & 10.567 & 2.7 & 0.0063 \\
\hline Dissolved oxygen & 0.083 & 0.048 & 1.7 & 0.0870 & 0.160 & 0.038 & 4.2 & $<0.0001$ \\
\hline Covariance parameters & $\theta_{1}$ & $\theta_{2}$ & & $\sigma^{2}$ & $\theta_{1}$ & $\theta_{2}$ & & \\
\hline Estimate & 0.280 & 0.084 & & 0.519 & 0.269 & 0.076 & & \\
\hline Likelihood ratio test & $d f=2$ & $X^{2}=88.3$ & & $<0.0001$ & $d f=2$ & $X^{2}=117.7$ & & 001 \\
\hline \multirow[t]{2}{*}{ Variable } & \multicolumn{4}{|c|}{ Species richness 1980-89 } & \multicolumn{4}{|c|}{ Species density 1980-89 } \\
\hline & coefficient & s.e. & $t$ & $P$ & coefficient & s.e. & $t$ & $P$ \\
\hline Intercept & 1.727 & 1.158 & 1.5 & 0.2744 & 0.554 & 0.914 & 0.6 & 0.6062 \\
\hline SST & -0.402 & 0.199 & -2.0 & 0.0436 & -0.150 & 0.158 & -1.0 & 0.3424 \\
\hline$(\mathrm{SST})^{2}$ & 0.038 & 0.012 & 3.3 & 0.0012 & 0.020 & 0.009 & 2.1 & 0.0334 \\
\hline$(\mathrm{SST})^{3}$ & -0.001 & 0.0002 & -3.7 & 0.0003 & 0.000 & 0.0002 & -2.5 & 0.0133 \\
\hline SST gradient & 28.695 & 14.936 & 1.9 & 0.0551 & 18.552 & 11.737 & 1.6 & 0.1144 \\
\hline Dissolved oxygen & 0.160 & 0.052 & 3.1 & 0.0022 & 0.108 & 0.043 & 2.5 & 0.0118 \\
\hline Covariance parameters & $\theta_{1}$ & $\theta_{2}$ & & $\sigma^{2}$ & $\theta_{1}$ & $\theta_{2}$ & & \\
\hline Estimate & 0.220 & 0.082 & & 0.529 & 0.188 & 0.071 & & \\
\hline Likelihood ratio test & $d f=2$ & $X^{2}=85.3$ & & $<0.0001$ & $d f=2$ & $X^{2}=132.7$ & & 001 \\
\hline \multirow[t]{2}{*}{ Variable } & \multicolumn{4}{|c|}{ Species richness 1990-99 } & \multicolumn{4}{|c|}{ Species density 1990-99 } \\
\hline & coefficient & s.e. & $t$ & $P$ & coefficient & s.e. & $t$ & $P$ \\
\hline Intercept & 1.291 & 0.852 & 1.5 & 0.2689 & 0.644 & 0.637 & 1.0 & 0.4184 \\
\hline SST & -0.427 & 0.152 & -2.8 & 0.0050 & -0.323 & 0.115 & -2.8 & 0.0050 \\
\hline$(\mathrm{SST})^{2}$ & 0.040 & 0.009 & 4.3 & $<0.0001$ & 0.030 & 0.007 & 4.2 & $<0.0001$ \\
\hline$(\mathrm{SST})^{3}$ & -0.001 & 0.0002 & -4.7 & $<0.0001$ & -0.001 & 0.0001 & -4.5 & $<0.0001$ \\
\hline SST gradient & 48.697 & 14.107 & 3.5 & 0.0006 & 34.613 & 10.136 & 3.4 & 0.0007 \\
\hline Dissolved oxygen & 0.181 & 0.050 & 3.6 & 0.0004 & 0.177 & 0.040 & 4.5 & $<0.0001$ \\
\hline Covariance parameters & $\theta_{1}$ & $\theta_{2}$ & & $\sigma^{2}$ & $\theta_{1}$ & $\theta_{2}$ & & \\
\hline Estimate & 0.241 & 0.086 & & 0.481 & 0.178 & 0.070 & & \\
\hline Likelihood ratio test & $d f=2$ & $X^{2}=69.2$ & & $<0.0001$ & $d f=2$ & $X^{2}=142.8$ & & 001 \\
\hline
\end{tabular}




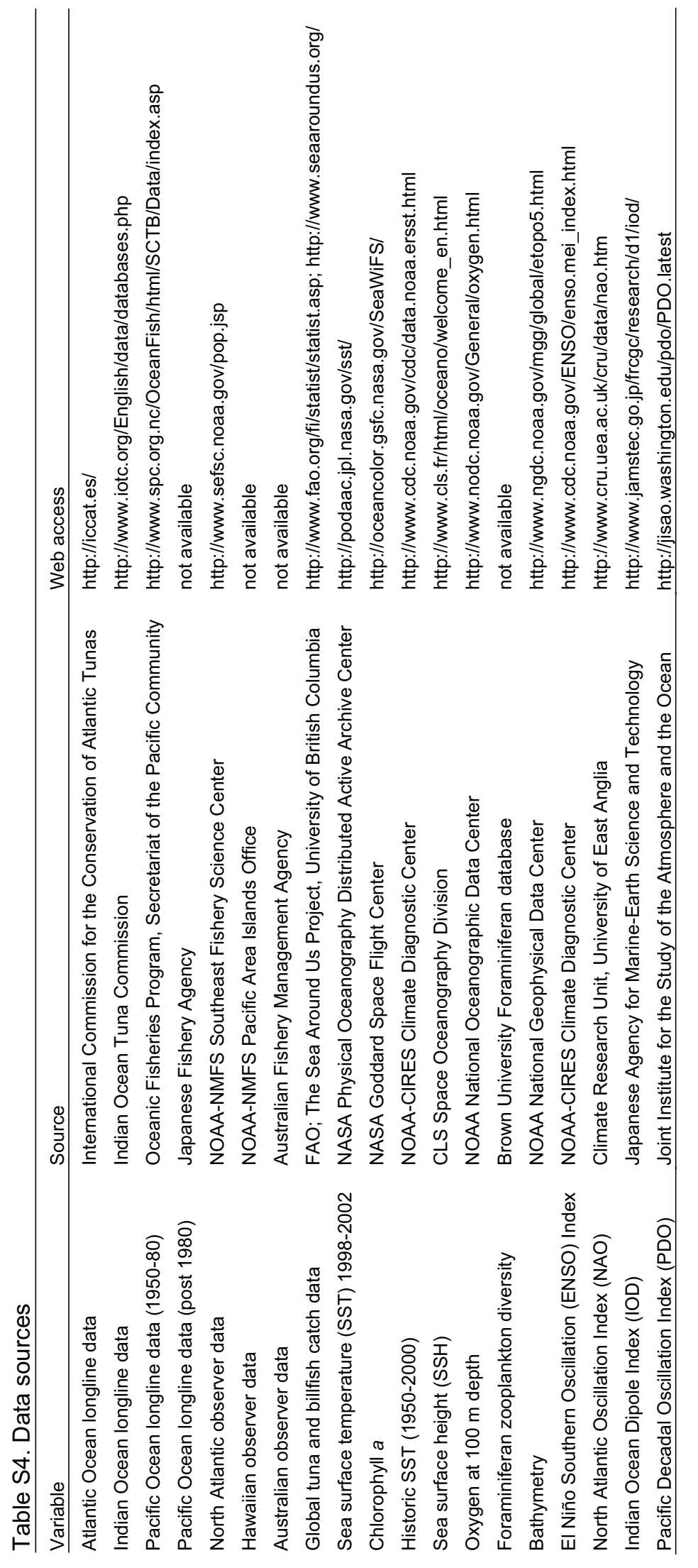



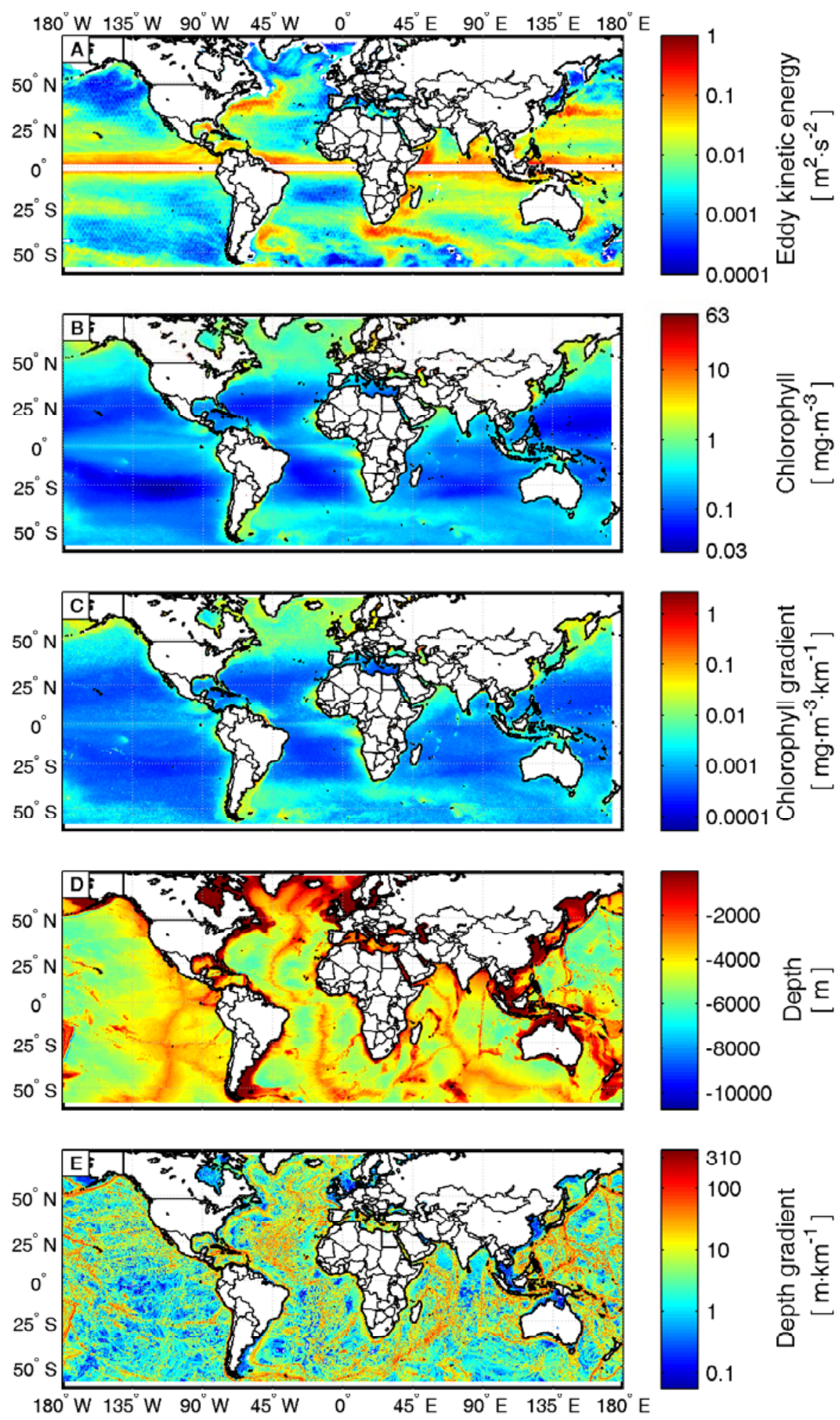

Fig. S1. Additional oceanographic variables used in the analysis. (A) eddy kinetic energy as derived from altimeter data, (B) mean chlorophyll a concentrations, (C) spatial chlorophyll a gradient, (D) depth, and $(\mathrm{E})$ spatial bathymetric gradient. 